\title{
USO DA VINHAÇA NO ABACAXIZEIRO EM SOLO DE BAIXO POTENCIAL DE PRODUÇÃO'
}

\author{
MIRALDA BUENO DE PAULA², FRANCISCO SANDRO RODRIGUES HOLANDA ${ }^{3}$, \\ HUGO ADELANDE MESQUITA ${ }^{4}$ e VÂNIA DÉA DE CARVALHO ${ }^{5}$
}

\begin{abstract}
RESUMO - O trabalho foi desenvolvido com o objetivo de avaliar a vinhaça como fonte de $\mathrm{K}$ para o abacaxizeiro (Ananas Comosus L.), em substituição ao $\mathrm{KCl}$, e seus efeitos sobre as características químicas do solo. O experimento foi instalado em um LV, textura argilosa, utilizando a cv. Smooth Cayenne. Os tratamentos constaram de quatro doses de vinhaça $\left(0-100-200-400 \mathrm{~m}^{3} / \mathrm{ha}\right)$, mais um tratamento adicional: $12 \mathrm{~g} /$ planta $\mathrm{K}_{2} \mathrm{O}(\mathrm{KCl})$. O delineamento experimental foi em blocos ao acaso, com três repetições. Verificou-se efeito significativo da vinhaça e do $\mathrm{KCl}$ sobre a produção. Com $400 \mathrm{~m}^{3} / \mathrm{ha}$ de vinhaça e $20,5 \mathrm{~g} /$ planta de $\mathrm{KCl}$, os rendimentos tiveram um acréscimo de $70 \%$ e $73 \%$, respectivamente, em relação à testemunha. $\mathrm{O}$ fornecimento de $\mathrm{K}$ elevou a porcentagem de acidez titulável total e sólidos solúveis totais nos frutos, porém não houve diferença significativa entre as fontes. Os teores foliares de $\mathrm{K}$ foram aumentados significativamente pela aplicação de vinhaça e de $\mathrm{KCl}$, e os teores de $\mathrm{Mg}$ decresceram. A aplicação de vinhaça contribuiu para aumento, no solo, dos teores dos cátions $\mathrm{K}, \mathrm{Ca}, \mathrm{Mg}$ e para a lixiviação de $\mathrm{K}$. Vinhaça e $\mathrm{KCl}$ elevaram a níveis adequados, para cultura, a porcentagem de $\mathrm{K}$ na soma de bases.
\end{abstract}

Termos para indexação: resíduo de destilaria, potássio, produção de abacaxi, Ananas comosus.

\section{STILLAGE APPLICATION FOR PINEAPPLE IN SOIL WITH LOW POTENTIAL OF YIELD}

ABSTRACT - An experiment was carried out in order to evaluate the stillage as K source for pineapple (Ananas Comosus L.) in comparison with $\mathrm{KCl}$, and the effects on chemical soil characteristics. The experiment was assessed in a Red Yellow Latosol with Smooth Cayenne cultivar. The treatments consisted of four application doses of stillage (0 - $\left.100-200-400 \mathrm{~m}^{3} / \mathrm{ha}\right)$ and of an additional treatment $\left(12 \mathrm{~g} /\right.$ plant $\left.\mathrm{K}_{2} \mathrm{O} \mathrm{KCl}\right)$. The design of the trial was in randomized blocks with three replications. Yields statistically responded to stillage and $\mathrm{KCl}$ and yields obtained with $400 \mathrm{~m}^{3} / \mathrm{h}$ of stillage and $20.5 \mathrm{~g}$ of $\mathrm{KCl}$ per plant were $70 \%$ and $73 \%$, respectivelly, over the control. Addition of $\mathrm{K}$ increased the content of tritratable acidity and total soluble solids in the fruit with no difference between the sources. Concentration of $\mathrm{K}$ in the leaves increased and $\mathrm{Mg}$ content decreased by stillage and $\mathrm{KCl}$ application. Addition of stillage in the soil increased $\mathrm{K}, \mathrm{Ca}, \mathrm{Mg}$ content and leached $\mathrm{K}$ to $20-40 \mathrm{~cm}$ depth. Stillage and $\mathrm{KCl}$ increased $\mathrm{K}$ to adequate levels in the bases sum.

Index terms: distillery residue, potassium, pineapple yield, Ananas comosus.

\section{INTRODUÇ̃̃O}

A cultura do abacaxi em Minas Gerais tem sido instalada em solos de baixa fertilidade. A adoção de

\footnotetext{
${ }^{1}$ Aceito para publicação em 11 de novembro de 1998.

${ }^{2}$ Enga agra ${ }^{\mathrm{a}}$, D.Sc., Centro Regional do Sul de Minas Gerais (CRSM)/EPAMIG, Caixa Postal 176, CEP 37200-000 Lavras, MG, Bolsista do CNPq. E-mail: bueno@ufla.br

${ }^{3}$ Eng. Agr., D.Sc., Prof. Visitante, Universidade Federal de Lavras (UFLA), Caixa Postal 37, CEP 37200-000 Lavras, MG.

${ }^{4}$ Eng. Agr., M.Sc., CRSM/EPAMIG.

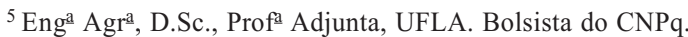

um sistema de manejo que eleve ou mantenha os teores de matéria orgânica é fundamental para a manutenção da fertilidade. Uma das propriedades físico-químicas mais importantes do solo é a sua capacidade de troca catiônica (CTC), e a CTC da matéria orgânica pode atingir valores maiores que os dos colóides minerais.

As pesquisas mostram a grande exigência do abacaxizeiro em certos elementos minerais. Paula et al. (1985) verificaram ser o K o nutriente absorvido em maior quantidade. Segundo Carvalho et al. (1994), este nutriente é também importante para a qualidade do fruto. 
$\mathrm{O} \mathrm{K}$ é fornecido às plantas combinado principalmente com o cloreto ou sulfato. Contudo, pode-se considerar como fonte alternativa a vinhaça. Segundo Glória \& Orlando Filho (1975) e Glória \& Santa Ana (1975), a composição da vinhaça é bastante variável e depende da matéria-prima usada, do tipo de destilado a ser obtido e do tipo de fermentação empregada. Normalmente, é rica em matéria orgânica e tem composição mineral variável, com predominância do $\mathrm{K}$ entre os cátions.

O uso de resíduos orgânicos da indústria canavieira na abacaxicultura tem sido pouco estudado. O presente trabalho teve como objetivo verificar a possibilidade de utilização da vinhaça como fonte de $\mathrm{K}$, em substituição ao $\mathrm{KCl}$, no rendimento e qualidade do abacaxizeiro, e os efeitos dela sobre as características químicas do solo.

\section{MATERIAL E MÉTODOS}

O trabalho foi instalado no município de Três Pontas, MG, em um Latossolo Vermelho-Amarelo (LV), textura argilosa. Em amostras de solos obtidas a uma profundidade de 0-20 cm, efetuaram-se as análises químicas e físicas, pelo método de Vettori (1969), modificado por Embrapa (1979). Os dados médios utilizados foram os seguintes: $\mathrm{pH}$ em $\mathrm{H}_{2} \mathrm{O}(5,2) ; \mathrm{P} \quad\left(1 \mathrm{mg} / \mathrm{dm}^{3}\right)$; $\mathrm{K}\left(46 \mathrm{mg} / \mathrm{dm}^{3}\right) ; \mathrm{Ca}\left(20 \mathrm{mmol}_{\mathrm{c}} / \mathrm{dm}^{3}\right) ; \mathrm{Mg}\left(8 \mathrm{mmol}_{\mathrm{c}} / \mathrm{dm}^{3}\right)$; $\mathrm{Al}\left(2 \mathrm{mmol}_{\mathrm{c}} / \mathrm{dm}^{3}\right) ; \mathrm{H}+\mathrm{Al}\left(7,3 \mathrm{mmol}_{\mathrm{c}} / \mathrm{dm}^{3}\right) ; \mathrm{S}(\mathrm{K}+\mathrm{Ca}+$ $\mathrm{Mg})=29,1 \mathrm{mmol}_{\mathrm{c}} / \mathrm{dm}^{3} ; \mathrm{T}(\mathrm{K}+\mathrm{Ca}+\mathrm{Mg}+\mathrm{H}+$ $\mathrm{Al})=36,4 \mathrm{mmol}_{\mathrm{c}} / \mathrm{dm}^{3} ; \mathrm{V}(100 \mathrm{~S} / \mathrm{T})=79,9 \%$; M.O. $\left(28 \mathrm{~g} / \mathrm{dm}^{3}\right)$; areia $\left(92 \mathrm{~g} / \mathrm{dm}^{3}\right)$; limo $\left(100 \mathrm{~g} / \mathrm{dm}^{3}\right)$; argila $\left(808 \mathrm{~g} / \mathrm{dm}^{3}\right)$. Os extratores utilizados foram: P e K (Mehlich-1); $\mathrm{Ca}, \mathrm{Mg}$ e $\mathrm{Al}(\mathrm{KCl} 1 \mathrm{~mol} / \mathrm{L}) ; \mathrm{H}+\mathrm{Al}$ $\left(\mathrm{Ca}\left(\mathrm{OA}_{\mathrm{c}}\right)_{2}\right)$.

Foi plantada, em fevereiro, a cultivar Smooth Cayenne, em linhas duplas, com espaçamento de 1,20 + 0,40 x $0,40 \mathrm{~m}$. Cada parcela foi constituída por 60 plantas, sendo 32 plantas úteis. As mudas foram do tipo "rebentões", selecionadas quanto ao tamanho, utilizando-se as que tinham cerca de $26 \mathrm{~cm}$, conforme recomendação de Reinhardt (1985). A indução floral foi feita com ethrel, 12 meses após o plantio. A adubação básica constou da aplicação de 3,0 g/planta de $\mathrm{P}_{2} \mathrm{O}_{5}$ (superfosfato simples) no plantio, $12 \mathrm{~g} /$ planta $\mathrm{N}$ (uréia) parcelada em três aplicações, a saber: $1 / 3$ no plantio, $1 / 3$ quatro meses após o plantio e $1 / 3$ doze meses após o plantio.

Os tratamentos constaram de quatro doses de vinhaça (0 - 100 - 200 - $400 \mathrm{~m}^{3} / \mathrm{ha}$ ), mais um tratamento adicional de $12 \mathrm{~g} /$ planta de $\mathrm{K}_{2} \mathrm{O}(\mathrm{KCl})$. A vinhaça foi aplicada por aspersão manual com um regador, 15 dias antes do plantio. $\mathrm{O} \mathrm{KCl}$ foi aplicado no plantio, segundo a recomendação de Samuels \& Gandia-Diaz (1958). Os resultados de composição química da vinhaça em $\mathrm{g} / \mathrm{L}$ foram: $\mathrm{K}_{2} \mathrm{O}(1,2)$; $\mathrm{CaO}(0,18) ; \mathrm{MgO}(0,088) ; \mathrm{pH}(4,3)$. O delineamento experimental foi em blocos ao acaso, com três repetições.

Foram realizadas avaliações sobre: a) rendimento e peso médio do fruto (peso do fruto sem coroa); b) teores de nutrientes na matéria seca, doze meses após o plantio, amostradas cinco folhas "D" por tratamento em cada repetição, conforme Manica et al. (1984). As amostras foliares foram postas a secar em estufa a $65^{\circ} \mathrm{C}$. O N foi determinado pelo método micro-Kjeldahl, e no extrato obtido por digestão nítrico-perclórica determinaram-se os teores de $\mathrm{P}$ por colorimetria; $\mathrm{K}$, por fotometria de chama; $\mathrm{Ca}, \mathrm{Mg}$ e micronutrientes, por espectrofotometria de absorção atômica, conforme Malavolta et al. (1989); c) características tecnológicas dos frutos: sólidos solúveis totais (SST), expresso em porcentagem, utilizando o refratômetro de ABBÉ; acidez titulável total (ATT), por titulação, com NaOH $0,1 \mathrm{~mol} / \mathrm{L}$; esta foi expressa em porcentagem de ácido cítrico; d) características químicas do solo: amostras do solo foram coletadas 15 dias após aplicação dos tratamentos, a 0-20 e 20-40 cm de profundidade.

\section{RESULTADOS E DISCUSSÃO}

Obteve-se efeito significativo da vinhaça e $\mathrm{KCl}$ como fontes de K, sobre a produção e peso médio dos frutos. Com $400 \mathrm{~m}^{3} /$ ha de vinhaça e $20,5 \mathrm{~g} /$ planta de $\mathrm{KCl}$, os rendimentos de frutos tiveram um acréscimo em torno de $70 \%$ e $73 \%$, respectivamente, em relação à testemunha. No abacaxizeiro, aumento do peso médio dos frutos e incrementos de produção decorrentes da adubação com $\mathrm{K}$ foram obtidos por outros autores (Iuchi, 1978; Reinhardt, 1980).

O modelo linear descreve a relação entre as doses de vinhaça, a produção e o peso médio dos frutos (Figs. 1 e 2). Outros autores também encontraram aumento de produção pela utilização da vinhaça. Berton et al. (1983) obtiveram aumento de produção de matéria seca do milho. Freire et al. (1983), obtiveram resultados positivos na cultura da mandioca. Paula et al. (1989) obtiveram aumento da produção na cultura da batata, e Paula et al. (1992) obtiveram maior produção e maior índice agroindustrial (Iai) na cultura da cebola pela utilização da vinhaça; porém, seu maior emprego foi na cultura da cana. 
Quanto às características tecnológicas avaliadas, verificou-se que a acidez (ATT) aumentou com o K fornecido pela vinhaça ou $\mathrm{KCl}$. Esses resultados concordam com os obtidos por Tay (1972) e Bezerra et al. (1981). Houve efeito significativo das doses de vinhaça sobre a acidez dos frutos (Fig. 3). As maiores porcentagens de acidez $(0,63$ e 0,64$)$ foram obtidas com $400 \mathrm{~m}^{3} /$ ha de vinhaça e $20,5 \mathrm{~g} /$ planta de $\mathrm{KCl}$, respectivamente, quando houve nas folhas um estreitamento da relação N/K. Reinhardt (1980) verificou que, na adubação, uma ampla relação N/K $(4,5: 1)$ causou redução na acidez do fruto. Dados obtidos por Iuchi (1978) mostram correlação negativa entre $\mathrm{N}$ e acidez. Uma alta relação $\mathrm{N}: \mathrm{K}$ pode

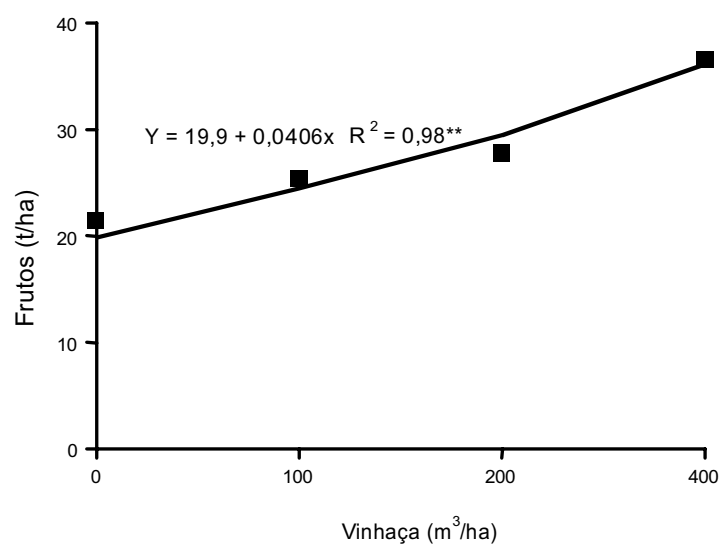

FIG. 1. Efeito das doses de vinhaça sobre a produção de frutos.

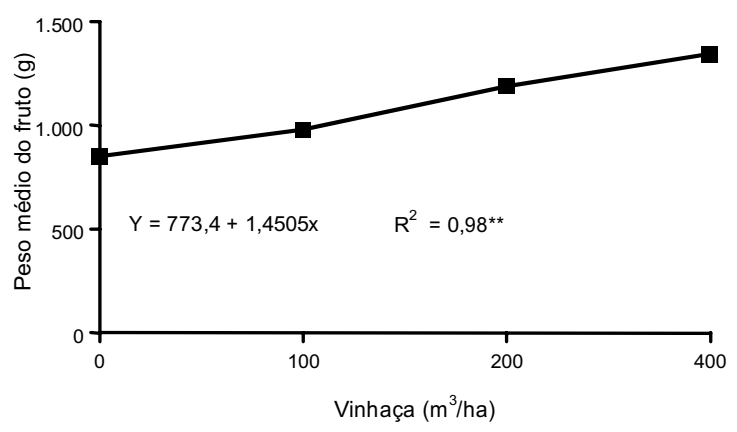

FIG. 2. Efeito das doses de vinhaça sobre o peso médio dos frutos. provocar maior turgescência dos frutos, e, indiretamente, a queda da acidez, em decorrência da diluição.

Observou-se aumento da porcentagem de sólidos solúveis totais (SST) com aplicação de $\mathrm{K}$ via vinhaça ou $\mathrm{KCl}$. Houve efeito significativo das doses de vinhaça sobre a porcentagem de SST (Fig. 3), e o valor de SST obtido pela aplicação de $\mathrm{KCl}$ foi de 18,1. Os dados são concordantes com os obtidos por Bezerra et al. (1981) e Paula et al. (1991). Haag et al. (1963) verificaram que a menor porcentagem de SST foi encontrada em plantas carentes de K.

Não houve diferença significativa entre as fontes de $\mathrm{K}$ (vinhaça e $\mathrm{KCl}$ ) sobre essas variáveis. Segundo Marchal et al. (1981), os efeitos das fontes de K sobre as características tecnológicas dos frutos dependem das condições climáticas, da época de aplicação e do estádio de desenvolvimento da planta. Deve-se considerar, também, que a sensibilidade das plantas a $\mathrm{Cl}$ depende também da dose de $\mathrm{K}$ e da cultivar.

Os teores foliares de $\mathrm{K}$ elevaram-se e os de $\mathrm{Mg}$ decresceram significativamente pela aplicação de vinhaça e de $\mathrm{KCl}$. O modelo linear descreve a relação entre doses de vinhaça e teores de $\mathrm{K}$ e $\mathrm{Mg}$ (Fig. 4). A aplicação de altas doses de K geralmente reduz os teores de $\mathrm{Ca}$ e $\mathrm{Mg}$, mas com relação ao abacaxizeiro esse efeito interfere mais na absorção de Mg. Alvarenga \& Couto, citados por Alvarenga (1981) e Paula et al. (1991), observaram que aplicação de altas doses de $\mathrm{K}$ reduziu o conteúdo de $\mathrm{Mg}$ nas folhas do abacaxizeiro. O decréscimo no conteúdo de $\mathrm{Mg}$ não afetou o rendimento, o que con-

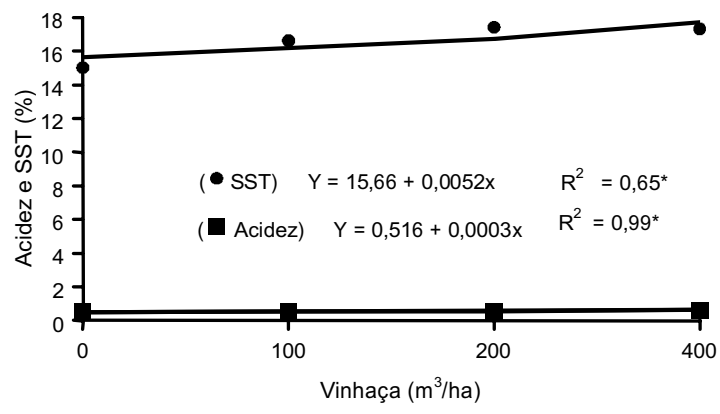

FIG. 3. Efeito das doses de vinhaça sobre acidez e sólidos solúveis totais (SST) dos frutos.

Pesq. agropec. bras., Brasília, v.34, n.7, p.1217-1222, jul. 1999 
corda com os dados obtidos por Hernandez-Medina (1964). Esse autor, verificou que respostas a Mg só ocorrem quando o teor foliar for inferior a $0,30 \%$ de $\mathrm{MgO}$.

Os teores dos demais nutrientes não foram influenciados pelos tratamentos e se encontravam dentro da faixa adequada conforme Malavolta et al. (1989), e seus valores médios são apresentados na Tabela 1.

Quanto às características químicas do solo, o efeito do $\mathrm{KCl}$ sobre o teor de $\mathrm{K}$ foi avaliado apenas na camada de 0-20 cm, obtendo-se uma concentração de $214 \mathrm{mg} / \mathrm{dm}^{3}$, que não diferiu significativamente da concentração obtida com $400 \mathrm{~m}^{3} /$ ha de vinhaça. Verificou-se, na camada de 0-20 e 20-40 cm, aumento nos teores de $\mathrm{K}$, proporcional às doses de vinhaça aplicadas (Fig. 5). Resultados semelhantes foram obtidos por Camargo et al. (1983) e Paula et al. (1989, 1992). A aplicação da vinhaça, provo-

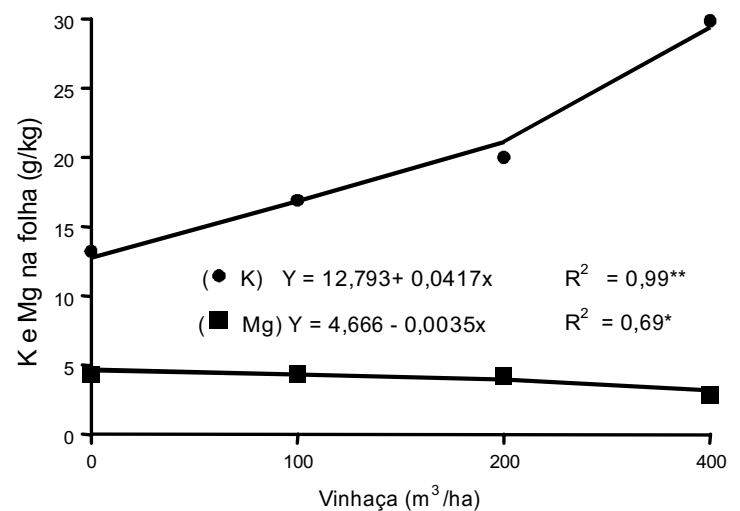

FIG. 4. Teores foliares de K e Mg do abacaxizeiro resultantes das doses de vinhaça. cando teores mais elevados desse nutriente no complexo sortivo, pode acarretar acréscimos de $\mathrm{K}$ também na solução do solo, e propicia, portanto, maior susceptibilidade à lixiviação, contribuindo, assim, para o aumento do $\mathrm{K}$ em profundidade. Contudo, os teores que permaneceram na camada arável $(0-20 \mathrm{~cm})$ foram superiores aos obtidos no solo original. A vinhaça elevou significativamente os teores de $\mathrm{Ca}$ e $\mathrm{Mg}$, à profundidade de 0-20 cm (Fig. 6), mas não alterou seus valores em profundidade (20-40 cm), e os valores médios de Ca e Mg nesta camada foram de 12 e $6 \mathrm{mmol}_{\mathrm{c}} / \mathrm{dm}^{3}$, respectivamente. Apesar de os valores absolutos dos cátions terem sofrido acréscimo generalizado, o aumento do $\mathrm{K}$ se deu em razão bem maior, provocando maior saturação de K na soma de bases ( $\mathrm{S}$ ) na camada de 0-20 cm. No solo original, a porcentagem de $\mathrm{K}$ em $\mathrm{S}$ foi de 4,22 , e ficou em torno de $15 \%$ na presença de $400 \mathrm{~m}^{3} /$ ha de vinhaça e de $\mathrm{KCl}$ (Tabela 2). Paula

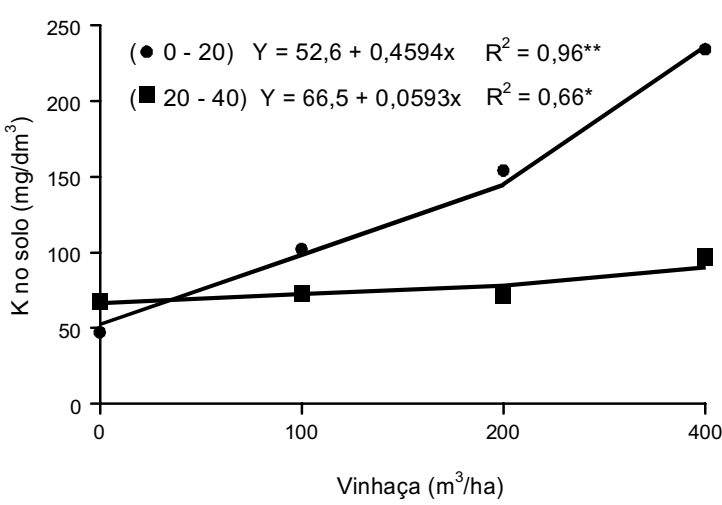

FIG. 5. Efeito das doses de vinhaça sobre os teores de $\mathrm{K}$ no solo à profundidade de $0-20$ e $20-40 \mathrm{~cm}$.

TABELA 1. Efeito das doses de vinhaça e do KCl no teor de nutrientes na matéria seca de folhas do abacaxizeiro.

\begin{tabular}{cccccccc}
\hline $\begin{array}{c}\text { Vinhaça } \\
\left(\mathrm{m}^{3} / \mathrm{ha}\right)\end{array}$ & $\mathrm{N}$ & $\mathrm{P}$ & $\mathrm{Ca}$ & $\mathrm{Cu}$ & $\mathrm{Fe}$ & $\mathrm{Mn}$ & $\mathrm{Zn}$ \\
\hline 0 & 22 & 2,3 & 4,2 & 10 & 290 & 678 & 9,5 \\
100 & 22 & 2,2 & 4,1 & 09 & 281 & 601 & 9,2 \\
200 & 21 & 2,2 & 4,3 & 12 & 276 & 695 & 9,4 \\
400 & 21 & 2,3 & 4,2 & 10 & 283 & 684 & 9,3 \\
$\mathrm{KCl}$ & 22 & 2,2 & 4,3 & 11 & 289 & 677 & 9,5 \\
\hline
\end{tabular}




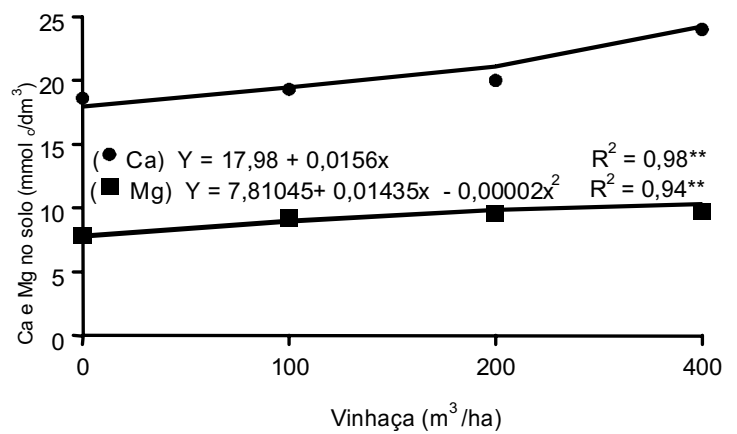

FIG. 6. Efeito das doses de vinhaça sobre os teores de Ca e Mg no solo, à profundidade de 0-20 cm.

TABELA 2. Efeito das doses de vinhaça sobre os teores de bases $(\mathrm{K}, \mathrm{Ca}, \mathrm{Mg})$ no solo $(0-20 \mathrm{~cm})$ e porcentagem (\%) de $K$ na soma de bases (S), de um Latossolo Vermelho (LV).

\begin{tabular}{cccccc}
\hline $\begin{array}{c}\text { Vinhaça } \\
\left(\mathrm{m}^{3} / \mathrm{ha}\right)\end{array}$ & \multicolumn{1}{c}{ K } & $\begin{array}{c}\mathrm{Ca} \\
\mathrm{Mg}\end{array}$ & $\mathrm{S}$ & $\mathrm{K}$ em S \\
\hline 0 & 1,18 & 18,6 & 8,0 & 27,7 & 4,22 \\
100 & 2,6 & 19,3 & 9,2 & 31,1 & 8,36 \\
200 & 3,9 & 20,0 & 9,7 & 33,6 & 11,60 \\
400 & 6,1 & 24,0 & 9,8 & 39,9 & 15,28 \\
$\mathrm{KCl}$ & 5,4 & 19,7 & 8,8 & 33,9 & 15,90 \\
\hline
\end{tabular}

et al. (1991) obtiveram, com o abacaxizeiro, cv. Pérola, produção máxima quando a participação do K na soma de bases foi de $20 \%$. No presente trabalho, o modelo que descreve a relação vinhaça x produção foi linear, e a mais alta saturação de K na soma de bases foi de $15 \%$, o que confirma os dados dos autores citados.

\section{CONCLUSÕES}

1. A vinhaça na dosagem adequada substitui o $\mathrm{KCl}$ como fonte de $\mathrm{K}$ para o abacaxizeiro, com aumento no rendimento de frutos.

2. Acidez e sólidos solúveis totais dos frutos aumentam com fornecimento de $\mathrm{K}$, independentemente da fonte: vinhaça ou $\mathrm{KCl}$.
3. A vinhaça e o $\mathrm{KCl}$ elevam a participação de $\mathrm{K}$ na soma de bases a níveis adequados à obtenção de maiores rendimentos.

\section{REFERÊNCIAS}

ALVARENGA, L.R. de. Nutrição mineral do abacaxizeiro. Informe Agropecuário, Belo Horizonte, v.7, n.74, p.18-24, 1981.

BERTON, R.S.; GERALDI, R.N.; RAIJ, B. van. Resíduos da indústria alcooleira como fonte de potássio em experimento em casa de vegetação. Revista Brasileira de Ciência do Solo, Campinas, v.7, n.1, p.61-64, 1983.

BEZERRA, J.E.F.; MAAZE, V.C.; SANTOS, V.F.; LEDERMAN, I.E. Efeito da adubação nitrogenada, fosfatada e potássica na produção e qualidade do abacaxi, cv. Smooth Cayenne. Revista Brasileira de Fruticultura, Recife, v.3, n. único, p.1-5, 1981.

CAMARGO, O.A. de; VALADARES, J.M.A. da S.; GERALDI, R.N. Características químicas e físicas de solo que recebeu vinhaça por longo tempo. Campinas: IAC, 1983. 30p. (IAC. Boletim técnico, 76)

CARVALHO, J.G.; OLIVEIRA JÚNIOR, J.P.; PAULA, M.B. de; BOTREL, N. Influência dos nutrientes na qualidade de frutos. Informe Agropecuário, Belo Horizonte, v.17, n.180, p.52-55, 1994.

EMBRAPA. Serviço Nacional de Levantamento e Conservação do Solo (Rio de Janeiro, RJ). Manual de métodos de análise de solo. Rio de Janeiro, 1979.

FREIRE, F.M.; ROCHA, B.V.; RIBEIRO, A.C.; FREIRE, J.C. Vinhoto como fertilizante na cultura da mandioca. Belo Horizonte: EPAMIG, 1983. Não paginado. (EPAMIG. Pesquisando, 76).

GLÓRIA, N.A. da; ORLANDO FILHO, J. Determinação de nitrogênio, fósforo e potássio em tecido vegetal e vinhaça por digestão sulfúrica. Brasil Açucareiro, Rio de Janeiro, v.88, n.4, p.298-304, 1975.

GLÓRIA, N.A. da; SANTA ANA, A.G. Métodos de análise de resíduos de usinas de açúcar e destilarias. Revista da Agricultura, Piracicaba, v.50, n.1/2, p.29-34, 1975.

HAAG, H.P.; ARZOLLA, S.; MELLO, A.F.; BRASIL SOBRINHO, M.O.C.; OLIVEIRA, E.R.; MALAVOLTA, E. Estudos sobre a alimentação

Pesq. agropec. bras., Brasília, v.34, n.7, p.1217-1222, jul. 1999 
mineral do abacaxi (Ananas comosus) Sch. Anais da Escola Superior de Agricultura "Luiz de Queiroz", v.20, p.33-40, 1963.

HERNANDEZ-MEDINA, E. Magnesium an important nutrient in pineapple production in a Bayamon sandy clay. Journal of Agriculture of University of Puerto Rico, v.48, p.17-24, 1964.

IUCHI, V.L. Efeito de sulfato de amônio, superfosfato simples e sulfato de potássio sobre algumas características da planta e qualidade do fruto do abacaxizeiro, Ananas comosus (L.) Merr., variedade Smooth Cayenne. Viçosa: UFV, 1978. 61p. Tese de Mestrado.

MALAVOLTA, E.; VITTI, G.C.; OLIVEIRA, S.A. de. Avaliação do estado nutricional das plantas: princípios e aplicações. Piracicaba: POTAFOS, 1989. $201 \mathrm{p}$.

MANICA, I.; PASSOS, L.P.; IUCHI, V.L.; DEFELIPO, B.V.; LIECHTEMBERG, L.A.; CONDE, A.R. Efeito de três doses de cloreto e sulfato de potássio na concentração de macronutrientes nas folhas do abacaxizeiro (Ananas comosus (L.) Merril) cultivar Smooth Cayenne em Visconde do Rio Branco, MG, Brasil. In: CONGRESSO BRASILEIRO DE FRUTICUlTURA, 7., 1984, Florianópolis. Anais.. Florianópolis: Sociedade Brasileira de Fruticultura, 1984. v.1, p.105-144.

MARCHAL, J.; PINON, A.; TEISSON, C. Effects de la forme d'engrais potassiques sur la qualité de l'ananas en Côte d'Ívoire. Fruits, Paris, v.36, n.12, p.737-743, 1981.

PAULA, M.B. de; CARVALHO, V.D. de; NOGUEIRA, F.D. Efeitos da vinhaça na produção e qualidade da cebola em solo de baixo potencial de produtividade. Pesquisa Agropecuária Brasileira, Brasília, v.27, n.3, p.389-393, mar. 1992
PAULA, M.B. de; CARVALHO, J.G. de; NOGUEIRA, F.D.; SILVA, C.R. de R. Exigências nutricionais do abacaxizeiro. Informe Agropecuário, Belo Horizonte, v.11, n.130, p.27-31, 1985.

PAULA, M.B. de; CARVALHO, V.D. de; NOGUEIRA, F.D.; SOUZA, L.F. da S. Efeito da calagem, potássio e nitrogênio na produção e qualidade do fruto do abacaxizeiro. Pesquisa Agropecuária Brasileira, Brasília, v.26, n.9, p.1337-1343, set. 1991.

PAULA, M.B. de; FONTES, P.C.R.; CARVALHO, V.D. de; NOGUEIRA, F.D. Utilização de vinhaça como fonte de potássio para a cultura da batata. Horticultura Brasileira, Brasília, v.7, n.2, p.6-8, 1989.

REINHARDT, D.H.R.C. Produção e qualidade do abacaxi Pérola em diferentes densidades de plantio e níveis de adubação NPK. Pesquisa Agropecuária Brasileira, Brasília, v.15, n.4, p.399-404, out. 1980.

REINHARDT, D.H.R.C. Propagação do abacaxi. Informe Agropecuário, Belo Horizonte, v.11, n.130, p.18-21, 1985.

SAMUELS, G.; GANDIA-DIAZ, H. Influence of the number of fertilizer applications on pineapple yields. Journal of Agriculture of University of Puerto Rico, San Jose, v.42, p.7-11, 1958.

TAY, T.H. Comparative study of the different types of fertilizers as sources of nitrogen, phosphorus and potassium in pineapple cultivation. Tropical Agriculture of Trinidad, v.49, p.51-59, 1972.

VETTORI, L. Métodos de análise de solo. Rio de Janeiro: Ministério da Agricultura. Equipe de Pedologia e Fertilidade do Solo, 1969. 24p. (Boletim técnico, 7). 